\title{
Conteúdo de molibdênio das sementes de feijoeiro em resposta a doses do micronutriente pulverizado sobre as plantas
}

\author{
Seed molybdenum content of common bean in response to rates of micronutrient sprayed \\ over the plants
}

\author{
Rogério Faria Vieira $^{\mathrm{I}}$ Luís Tarcísio Salgado ${ }^{\mathrm{I}}$ André Assis Pires ${ }^{\mathrm{II}}$ Gilmar Silvério Rocha ${ }^{\mathrm{III}}$
}

\section{- NOTA-}

\section{RESUMO}

Os objetivos deste estudo foram verificar se altas doses de Mo aplicado na folhagem são tóxicas ao feijoeiro $e$ determinar o conteúdo de Mo das sementes em resposta a essas doses. Para tanto, foi conduzido um ensaio em Coimbra, Minas Gerais (MG), em solo com pH de 5,2, onde testaram-se as doses de 0, 90, 1000, 2000, 3000 e 4000 $\mathrm{ha}^{-1}$ de Mo. A dose de 90 foi aplicada na fase V4 dos feijoeiros. A dose de 1000 foi distribuída de três modos: toda ela na fase V4; 1/2 na V4 e 1/2 na R5; e 1/4 na V4, 1/4 na R5, 1/4 na R6, 1/4 na R7. As doses de 2000, 3000 e 4000 foram divididas em quatro partes iguais e aplicadas nas fases V4, R5, R6 e R7. As doses de Mo não influenciaram a produtividade de grãos, a massa de semente $e$ a germinação das sementes, o que indica que o Mo, até a dose de $4000 \mathrm{~kg} \mathrm{ha}^{-1}$, não é tóxico ao feijoeiro. O conteúdo de Mo da semente aumentou de 0,007 a 6,961̀̀ g com o incremento das doses de Mo.

Palavras-chave: Phaseolus vulgaris, teor de molibdênio, germinação das sementes.

\section{ABSTRACT}

The objectives of this research were to verify if high Mo rates sprayed on foliage are toxic to common bean and to determine seed Mo content in response to these rates. One experiment was carried out in Coimbra, $M G$, in soil with $\mathrm{pH}$ of 5.2, where it was tested the rates of 0,90,1000, 2000, 3000, and $4000 \mathrm{~g} \mathrm{ha}^{-1}$ of Mo. The rate of 90 was applied at V4 phase of common bean. The rate of 1000 was applied in three ways: all at V4 phase; $1 / 2$ at V4 and $1 / 2$ at R5; and $1 / 4$ at V4, 1/4 at R5, $1 / 4$ at $R 6,1 / 4$ at $R 7$. The rates of 2000, 3000, and 4000 were divided in four equal portions and sprayed at V4, R5, R6, and
$R 7$ phases. Mo rates had no effect on seed yield, seed mass and seed germination, indicating that Mo rates as high as $4000 \mathrm{~g}$ $\mathrm{ha}^{-1}$ is not toxic to common bean. Mo content in seed increased from 0.007 to 6.961ìg with increasing doses of Mo rates.

Key words: Phaseolus vulgaris, Mo concentration, seed germination.

O molibdênio (Mo) é constituinte das enzimas redutase do nitrato e da nitrogenase. Leguminosas que dependem da fixação simbiótica do $\mathrm{N}_{2}$ para a aquisição de $\mathrm{N}$ requerem mais Mo que plantas adubadas com nitrogênio (PARKER \& HARRIS, 1977).

Estudos conduzidos na Zona da Mata de Minas Gerais, em solos com deficiência de N e Mo, demonstram que a adubação molíbdica pode "substituir" a adubação nitrogenada em cobertura (VIEIRA et al., 1992; AMANE et al., 1999) para a obtenção de produtividades de até $3000 \mathrm{~kg} \mathrm{ha}^{-1}$ de feijão (VIEIRA et al., 2005). Nesses casos, o Mo foi aplicado na folhagem, na forma de solução, na fase V4 da leguminosa (VIEIRA et al., 1992; AMANE et al., 1999), ou fornecido por sementes com alto conteúdo desse micronutriente (VIEIRA et al., 2005). Nessa região, as maiores produtividades de feijão são alcançadas com a aplicação na folhagem de doses entre 70 e $100 \mathrm{~g} \mathrm{ha}^{-1}$ de Mo (BERGER et al., 1996; AMANE et al., 1999). A adubação molíbdica aumenta muito a atividade da

IEmpresa de Pesquisa Agropecuária de Minas Gerais (Epamig), Vila Gianetti, casa 47, CP 216, 36570-000, Viçosa, MG, Brasil

E-mail: rfvieira@epamig.br. *Autor para correspondência.

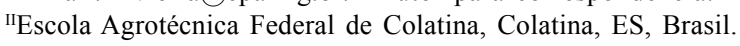

IIIPrograma de Pós-graduação em Fitotecnia, Universidade Federal de Viçosa (UFV), Viçosa, MG, Brasil 
nitrogenase e prolonga o período de alta atividade da redutase do nitrato (VIEIRA et al., 1998). Logo, a adubação molíbdica pode ser alternativa barata à adubação nitrogenada em cobertura e bem menos poluente que esta.

Apesar das respostas do feijão à adubação com Mo, muitos agricultores não têm acesso a essa tecnologia, quer por ignorá-la, quer por não haver disponibilidade do fertilizante no comércio local. Uma das opções para resolver esse problema é disponibilizar-lhes sementes com alto conteúdo de Mo (JACOB-NETO \& ROSSETTO, 1998; VIEIRA et al., 2005). Para tanto, é necessário aplicar altas doses de adubo molíbdico nos feijoeiros para que se colham sementes com alto conteúdo do micronutriente. Estudos demonstraram que altas doses de Mo (até $2550 \mathrm{~g} \mathrm{ha}^{-1}$ ) aplicados na folhagem não são tóxicas aos feijoeiros (VIEIRA et al., 2005; LEITE et al., 2007).

Os objetivos deste estudo foram verificar se a pulverização com doses de até $4000 \mathrm{~g} \mathrm{ha}^{-1}$ de Mo são tóxicas aos feijoeiros e determinar o conteúdo de Mo da semente em resposta a essas doses.

Foi conduzido um experimento em área da Universidade Federal de Viçosa, em Coimbra, Zona da Mata de Minas Gerais, entre maio e setembro de 2004. A análise química do solo (Argissolo VermelhoAmarelo, fase terraço) da camada de 0 a $20 \mathrm{~cm}$ forneceu os seguintes resultados: $\mathrm{pH}$ em água de 5,2; matéria orgânica de 3,46dag kg-1; CTC em pH de 7,0 e 7,42 de cmol dm ${ }^{3}$; V de 42\%; P (Mehlich 1) de $8 \mathrm{mg} \mathrm{dm}^{-3}$; K de $88 \mathrm{mg} \mathrm{dm}^{-3}$; Al de $0,1 \mathrm{cmol} \mathrm{dm}^{-3} ;$ Ca de $2,1 \mathrm{cmol} \mathrm{dm}^{-3} ; \mathrm{e}$ $\mathrm{Mg}$ de $0,8 \mathrm{cmol}_{\mathrm{c}} \mathrm{dm}^{-3}$. As doses, em $\mathrm{g} \mathrm{ha}^{-1}$, de Mo empregadas foram: 0, 90, 1000, 2000, 3000 e 4000. A dose de $90 \mathrm{~g} \mathrm{ha}^{-1}$ foi aplicada aos 30 dias após a emergência (DAE) (fase V4 dos feijoeiros). A dose de $1000 \mathrm{~g} \mathrm{ha}^{-1}$ foi distribuída de três modos: toda ela aos 30DAE; $1 / 2$ aos 30DAE e 1/2 aos 44DAE (fase R5); e 1/4 aos 30DAE, $1 / 4$ aos 44DAE, $1 / 4$ aos 58DAE (fase R6) e $1 / 4$ aos 71DAE (fase R7). As doses de 2000, 3000 e 4000g $\mathrm{ha}^{-1}$ de Mo foram divididas em quatro partes iguais e aplicadas aos 30, 44, 58 e 71DAE. Logo, foram avaliados oito tratamentos de Mo.

O delineamento foi em blocos ao acaso, com cinco repetições. Cada parcela constou de seis fileiras de $4 \mathrm{~m}$ de comprimento, espaçadas de $0,5 \mathrm{~m}$. Foram distribuídas 15 sementes por metro da cultivar 'Pérola', tipo carioca. Na colheita, eliminaram-se as duas fileiras laterais mais $0,5 \mathrm{~m}$ das extremidades das fileiras centrais. Portanto, a área útil foi de $6 \mathrm{~m}^{2}$. As sementes usadas no plantio foram colhidas de plantas não adubadas com Mo na Estação Experimental de Coimbra e tinham 2,055ì $\mathrm{g} \mathrm{g}^{-1}$ de Mo (equivalente a 0,462ì g de Mo por semente). Na adubação de plantio, foram usados $700 \mathrm{~kg}$ $\mathrm{ha}^{-1}$ do formulado comercial 4-14-8 (N- $\left.\mathrm{P}_{2} \mathrm{O}_{5}-\mathrm{K}_{2} \mathrm{O}\right)$. Em cobertura, aos 17DAE, foram distribuídos $200 \mathrm{~kg}$ $\mathrm{ha}^{-1}$ de sulfato de amônio em filete ao longo das fileiras. A distribuição de solução de molibdato de sódio sobre as plantas foi feita com pulverizar costal de $\mathrm{CO}_{2}$, equipado com bico XR 11002 e vazão equivalente a $251 \mathrm{~L} \mathrm{ha}^{-1}$. Um plástico com $1 \mathrm{~m}$ de largura foi estendido entre as parcelas vizinhas às tratadas para evitar que a deriva as atingisse.

As plantas daninhas foram controladas com os herbicidas fomesafen + fluazifop-p-butil $(0,25+0,20 \mathrm{~L}$ $\mathrm{ha}^{-1}$ ) aplicados em pós-emergência. Pragas como cigarrinha-verde (Empoasca kraemeri) e vaguinha (Diabrotica speciosa) foram controladas com o inseticida monocrotophos $\left(0,3 \mathrm{~L} \mathrm{ha} \mathrm{h}^{-1}\right)$. $\mathrm{O}$ controle de doenças foliares foi realizado, preventivamente, com o fungicida azoxystrobin $\left(50 \mathrm{~g} \mathrm{ha}^{-1}\right)$ aos 19,38 e 52DAE. As plantas foram irrigadas por aspersão, semanalmente, com uma lâmina de aproximadamente $50 \mathrm{~mm}$ de água. Os feijoeiros foram colhidos no estádio R9 (80\% das vagens amarelas ou secas e $30 \%$ das folhas ainda verdes).

Foram obtidos os seguintes dados: produtividade de grãos com $11 \%$ de água, massa de 100 sementes, teor e conteúdo de Mo na semente e germinação das sementes. A massa de 100 sementes correspondeu à média de três amostras de sementes colhidas de cada parcela. O teor de Mo nas sementes foi determinado colorimetricamente conforme metodologia descrita por VIEIRA et al. (2005). O conteúdo de Mo em uma semente foi obtido pelo produto entre o teor de Mo na semente e a massa de uma semente seca. O teste de germinação foi realizado em rolo de papel germitest, na temperatura de $25 \pm 1^{\circ} \mathrm{C}$, com três subamostras de 50 sementes por repetição. As sementes foram umedecidas com água deionizada, em quantidade equivalente a 2,5 vezes a sua massa. As avaliações foram feitas aos cinco e nove dias após o início do teste, de acordo com as Regras de Análise de Semente (BRASIL, 1992). O resultado do total de plântulas normais foi expresso em percentagem.

Os dados foram submetidos à análise de variância, e as médias foram comparadas pelo teste de Tukey, a 5\% de probabilidade.

Não houve efeito significativo das doses de Mo na produtividade de grãos, na germinação das sementes e na massa de 100 sementes (Tabela 1). Em estudo de campo, não houve efeito significativo das doses de zero a $1440 \mathrm{~g} \mathrm{ha}^{-1}$ de Mo aplicado na folhagem sobre a produtividade de feijão e a massa de 100 sementes (VIEIRA et al., 2005). Em ambos os estudos, a aplicação de $\mathrm{N}$ em cobertura, além do $\mathrm{N}$ distribuído na adubação de plantio, explica essa falta de resposta 
Tabela 1 - Produtividade de grãos e algumas características de sementes de feijão em resposta a doses de molibdênio em Coimbra, MG, 2004.

\begin{tabular}{|c|c|c|c|c|c|}
\hline Doses de $\mathrm{Mo}^{(1)}$ & Produtividade & $\begin{array}{l}\text { Germinação das } \\
\text { sementes }\end{array}$ & $\begin{array}{c}\text { Massa de } 100 \\
\text { sementes }\end{array}$ & $\begin{array}{l}\text { Teor de Mo na } \\
\text { semente }\end{array}$ & Conteúdo de $\mathrm{Mo}^{(2)}$ \\
\hline $\mathrm{g} \mathrm{ha}^{-1}$ & $\mathrm{~kg} \mathrm{ha}^{-1}$ & $\%$ & $\mathrm{~g}$ & $\mathrm{mg} \mathrm{kg}^{-1}$ & $\mu \mathrm{g}$ por semente \\
\hline $\mathrm{T} 1$ & $1732 \pm 91^{(3)}$ & $93,6 \pm 4,3$ & $22,38 \pm 1,05$ & $0,03 \pm 0,03 \mathrm{e}$ & $0,007 \pm 0,001 \mathrm{~d}$ \\
\hline $\mathrm{T} 2$ & $1783 \pm 252$ & $93,3 \pm 3,9$ & $23,08 \pm 1,12$ & $1,20 \pm 0,24 \mathrm{e}$ & $0,248 \pm 0,057 \mathrm{~d}$ \\
\hline $\mathrm{T} 3$ & $1840 \pm 202$ & $88,5 \pm 11,2$ & $22,92 \pm 0,74$ & $12,84 \pm 1,09 \mathrm{~d}$ & $2,615 \pm 0,172 \mathrm{c}$ \\
\hline $\mathrm{T} 4$ & $1880 \pm 254$ & $92,5 \pm 8,1$ & $23,08 \pm 0,83$ & $13,13 \pm 2,50 \mathrm{~d}$ & $2,700 \pm 0,542 \mathrm{c}$ \\
\hline T5 & $1795 \pm 214$ & $92,2 \pm 9,4$ & $23,04 \pm 0,78$ & $17,78 \pm 3,81 \mathrm{~cd}$ & $3,639 \pm 0,751 \mathrm{bc}$ \\
\hline $\mathrm{T} 6$ & $1778 \pm 158$ & $92,5 \pm 5,4$ & $23,18 \pm 0,82$ & $25,29 \pm 4,43 \mathrm{bc}$ & $5,236 \pm 1,033 a b$ \\
\hline $\mathrm{T} 7$ & $1810 \pm 107$ & $87,7 \pm 7,1$ & $23,10 \pm 1,45$ & $30,25 \pm 3,88 \mathrm{ab}$ & $6,212 \pm 0,836 \mathrm{a}$ \\
\hline $\mathrm{T} 8$ & $1884 \pm 239$ & $88,0 \pm 6,9$ & $22,90 \pm 0,69$ & $34,19 \pm 9,22 \mathrm{a}$ & $6,961 \pm 1,844 \mathrm{a}$ \\
\hline Média & 1813 & 91,1 & 22,96 & 16,78 & 3,452 \\
\hline
\end{tabular}

(1) As plantas foram adubadas com $\mathrm{N}$ no plantio $\left(28 \mathrm{~kg} \mathrm{ha}^{-1}\right)$ e em cobertura $\left(40 \mathrm{~kg} \mathrm{ha}^{-1}\right)$ : $\mathrm{T} 1=$ testemunha sem Mo, $\mathrm{T} 2=90 \mathrm{~g}$ ha ${ }^{-1}$ de $\mathrm{Mo}$ aos 30DAE; T3 = 1000 aos 30DAE; T4 = 500+500 aos 30 e 44DAE; T5 = 250+250+250+250 aos 30, 44, 58, 71DAE; T6 =500+500+500+500 aos 30, 44, 58, 71DAE; T7 = 750+750+750+750 aos 30, 44, 58, 71DAE; e T8 = 1000+1000+1000+1000 aos 30, 44, 58, 71DAE. (2) Calculado pela multiplicação da massa de uma semente seca pelo teor de Mo na semente. As médias seguidas da mesma letra não apresentam diferença significativa pelo teste de Tukey, a 5\% de probabilidade. Variáveis cujas médias não são seguidas de letras não foram influenciadas significativamente pelos tratamentos. ${ }^{(3)}$ Média de cinco repetições \pm desvio padrão.

do feijoeiro ao Mo. Sem aplicação de N em cobertura, as produtividades, em resposta a sete doses de Mo (de 0 a $2560 \mathrm{~g} \mathrm{ha}^{-1}$ ), se ajustaram ao modelo exponencial, com a dose ótima de Mo estimada em $255 \mathrm{~g} \mathrm{ha}^{-1}$ (LEITE et al., 2007). Nesse estudo de campo, as sementes produzidas com adubação molíbdica tiveram maior massa que as produzidas sem Mo. Quanto à germinação das sementes, os resultados deste estudo foram diferentes dos obtidos por BRODRICK et al. (1992), os quais verificaram que sementes produzidas em meio com deficiência de Mo, em casa de vegetação, apresentaram percentagem de germinação mais baixa que as sementes com alto conteúdo de Mo.

O conteúdo de Mo da semente aumentou de $0,007 \mu \mathrm{g}$ de Mo (testemunha que não recebeu Mo) para $6,961 \mu \mathrm{g}$ de Mo (4000g ha ${ }^{-1}$ de Mo), ou seja, houve um incremento de 994 vezes no conteúdo de Mo da semente (Tabela 1). O uso de sementes com alto conteúdo de Mo não prejudicou a nodulação da soja, diferentemente do que ocorreu quando as sementes foram tratadas com uma fonte de Mo (MILANI et al., 2008).

Não houve diferença significativa entre as doses de 2000, 3000 e 4000 $\mathrm{g} \mathrm{ha}^{-1}$ de Mo no conteúdo de Mo da semente. Atingiu-se, com a dose de $4000 \mathrm{~g} \mathrm{ha}^{-1}$ de Mo, quase o dobro do nível crítico (3,5ì g Mo por semente) sugerido por JACOB-NETO \& ROSSETTO (1998) como adequado para a auto-suficiência dos feijoeiros nesse micronutriente. Em solo com $\mathrm{pH}$ em água de 5,7, o conteúdo de Mo da cultivar 'Pérola' variou de 0,096 a 1,272ì g Mo por semente (aumento de 13,2 vezes), quando as doses de Mo variaram de zero a $1440 \mathrm{~g} \mathrm{ha}^{-1}$ (VIEIRA et al., 2005). Há relato na literatura de conteúdo na semente tão alto quanto 13,21 ì Mo em cultivar com massa de semente de $0,41 \mathrm{~g}$ (BRODRICK et al., 1995). O parcelamento da dose de $1000 \mathrm{~g} \mathrm{ha}^{-1} \mathrm{de}$ Mo não teve efeito significativo sobre o conteúdo de Mo da semente (Tabela 1). No entanto, em estudo também conduzido em Coimbra, houve maior conteúdo de Mo na semente com a aplicação parcelada (aos 17, 21, 27 e 32DAE) da dose de $1440 \mathrm{~g} \mathrm{ha}^{-1}$, em relação ao não parcelamento ou à aplicação de metade aos $17 \mathrm{e}$ metade aos 23DAE (VIEIRA et al., 2005). Em soja, o efeito do parcelamento das doses de Mo no conteúdo desse micronutriente na semente também pode ser neutro (MILANI et al., 2008) ou positivo (CAMPO et al., 2009). Esses resultados indicam que o efeito do parcelamento da adubação molíbdica no conteúdo de Mo da semente precisa ser mais estudado.

Conclui-se que doses tão altas quanto $4 \mathrm{~kg}$ $\mathrm{ha}^{-1}$ de Mo não são tóxicas quando pulverizadas nos feijoeiros e que é possível atingir 6,961 $\mu \mathrm{g}$ de Mo por semente com essa dose do micronutriente, sem haver prejuízos à germinação.

\section{AGRADECIMENTOS}

À Fundação de Amparo à Pesquisa do Estado de Minas Gerais (Fapemig), pelo apoio financeiro a este estudo.

Ciência Rural, v.40, n.3, mar, 2010. 


\section{REFERÊNCIAS}

AMANE, M.I.V. et al. Adubação nitrogenada e molíbdica da cultura do feijão na Zona da Mata de Minas Gerais. Revista Brasileira de Ciência do Solo, Viçosa, v.23, n.4, p.643650, 1999. Disponível em: ४ttp://sbcs.solos.ufv.br/solos/ revistas/v23n3a18.pdf>. Acesso em: 16 dez. 2009.

BERGER, P.G. et al. Efeitos de doses e épocas de aplicação do molibdênio sobre a cultura do feijão. Pesquisa Agropecuária Brasileira, Brasília, v.31, n.7, p.473-490, 1996. Disponível em: $<$ http://www.scielo.br/scieloOrg/php/reflinks.php?refpid=S0100 $8358200800010002500010 \& \mathrm{p}$ i d = S 0100 $83582008000100025 \& \operatorname{lng}=\mathrm{en}>$. Acesso em: 16 dez. 2009.

BRASIL. Ministério da Agricultura e Reforma Agrária. Regras para análise de sementes. Brasília: SNDA/DNDV/CLAV, 1992. 356p.

BRODRICK, S.J. et al. Molybdenum reserves of seed, and growth and $\mathrm{N}_{2}$ fixation by Phaseolus vulgaris L. Biology and Fertility of Soils, Heidelberg, v.13, n.1, p.39-44, 1992.

BRODRICK, S.J. et al. Seed analysis as a mean of identifying micronutrient deficiencies of Phaseolus vulgaris L. in the tropics. Tropical Agriculture, Trinidad, v.72, n.4, p.277284, 1995.

CAMPO, R.J. et al. Molybdenum-enriched soybean seeds enhance $\mathrm{N}$ accumulation, seed yield, and seed protein content in Brazil. Field Crops Research, Amsterdam, v.110, n.3, p.219-224, 2009. Disponível em: <http://www.sciencedirect.com/ science?_ob=ArticleURL\&_udi=B6T6M-4TSC3Y 4 $1 \&$ user $=687369 \&$ rdoc $=1 \&$ fmt $=\&$ orig $=$ search $\&$ s or $\mathrm{t}=\mathrm{d} \&$ docanchor $=\& \mathrm{view}=\overline{\mathrm{c}} \&$ _searchStrId $=1137 \overline{85}$ $7127 \&$ rerunOrigin $=$ scholar.google \& acct $=\mathrm{C} 000037901 \&$ version $=$ $1 \&$ u r l Version $=0 \&$ us e ri d $=687369 \&$ m d 5 $=$ afe $17 \mathrm{e} 37 \mathrm{~d} 94 \mathrm{ad} 897871 \mathrm{c} 2 \mathrm{a} 56 \overline{0} 2 \mathrm{dccca} 9>$. Acesso em: $16 \mathrm{dez}$ 2009. doi: 10.1016/j.fcr.2008.09.001.
JACOB-NETO, J.; ROSSETTO, C.A.V. Concentração de nutrientes nas sementes: o papel do molibdênio. Floresta e Ambiente, Seropédica, v.5, n.1, p.171-183, 1998.

LEITE, U.T. et al. Rendimento de grãos e componentes de rendimento do feijoeiro em função da aplicação foliar de doses crescentes de molibdênio. Acta Scientiarum Agronomy, Maringá, v.29, n.1, p.113-120, 2007. Disponível em: <http:/ /periodicos.uem.br/ojs/index.php/ActaSciAgron/article/view/ 74>. Acesso em: 16 dez. 2009.

MILANI, G.L. et al. Nodulação e desenvolvimento de plantas oriundas de sementes de soja com altos teores de molibdênio. Revista Brasileira de Sementes, Pelotas, v.30, n.2, p.1927, 2008. Disponível em: <http://www.scielo.br/pdf/rbs/v30n2/ a03v30n2.pdf>. Acesso em: 16 dez. 2009. doi: 10.1590/S010131222008000200003 .

PARKER, M.B.; HARRIS, H.B. Yield and leaf nitrogen of nodulating and non-nodulating soybeans as affected by nitrogen and molybdenum. Agronomy Journal, Madison, v.69, n.4, p.551-554, 1977.

VIEIRA, C. et al. Adubação nitrogenada e molíbdica na cultura do feijão. Revista de Agricultura, Piracicaba, v.67, n.2, p.117-124, 1992 .

VIEIRA, R.F. et al. Foliar application of molybdenum in common beans. I. Nitrogenase and reductase activities in a soil of high fertility. Journal of Plant Nutrition, Philadelphia, v. 21, n.1, p.169-180, 1998.

VIEIRA, R.F. et al. Performance of common bean using seeds harvested from plants fertilized with high rates of molybdenum. Journal of Plant Nutrition, Philadelphia, v.28, n.2, p.363$377,2005$. 